\title{
Impact of Design Factors of Disc Brake Rotor on Braking Performance
}

\author{
Atharva R Kulkarni \\ Department of Automotive Engineering \\ Vellore Institute of Technology \\ Vellore, India
}

\author{
Rohan Mahale \\ Department of Automotive Engineering \\ Vellore Institute of Technology \\ Vellore, India
}

\begin{abstract}
This study proposes to analyse structural stability and cooling characteristics of different brake rotor with varying geometries. SOLIDWORKS 2019 and ANSYS 19.3 Workbench was used for modelling and emulation of rotor operating characteristics. FEA approach was considered to find out the total deformation and temperature gradient for a single stop braking scenario. During braking operation, large amount of heat generation which has to be managed efficiently to keep the braking components in optimal operating temperature range. Failing to cool the rotor can cause excessive thermo-mechanical stresses which in turn causing brake fade, warping, cracking and judder in disc and thereby reducing lifespan. For avoiding these unnecessary failures, we have varied several design parameters of disc brake rotor by adding features rounded slots, drilled holes and so on. Several intrinsic material properties like surface topography, corrosion resistance and specific heat capacity also influence its durability and thermal performance to a large extent. The results give an idea how the different cooling structures affect the disc brake performance.
\end{abstract}

Keywords- ANSYS; Brake Rotor design; Cooling performance; FEA; Material selection; Thermo-Mechanical analysis

\section{INTRODUCTION}

Disc Brake Rotors are being engineered in a way such that they are durable, lighter and self-cleaning which could help the braking system to work more efficiently and give protective layering to the wheels and tires. These rotors provide a friction surface for brake callipers which are actuated by hydraulic mechanism. Disc brake rotors differ in design geometry for varying absorption and dissipation of heat. Disc Brake rotors are better at managing heat and perform better in wet conditions too. They also have more stopping power and shorter stopping distance than drum brakes. These are less prone to grabbing or pulling and are easier to service. Big size disc brake rotors are more efficient in absorbing and dissipating heat than small disc brake rotors. But due to its more weight it can affect the fuel efficiency and vehicle performance. Today, they are downsized to small disc brake rotors to reduce weight. The Disc brakes run quite hotter than drum brakes and thus require a better design and mechanism for cooling to keep the braking temperature within the safe limits.

There are currently different types of Disc Brake Rotors used in light weight vehicles for effective braking performance and thermal cooling which are better than that of drum brakes. These disc brake rotors have variations in its design which are used according to the vehicle requirements that includes the low-performance and high-performance vehicles. Due to its design variations, they indeed affect the manufacturing and machining costs. Today, there are many aftermarket performance disc brakes rotors available which provide much better cooling and ventilation. Table 1.0 shows disc brake rotors which have different variations in its design.

TABLE I. TYPES AND APPLICATION OF BRAKE ROTOR

\begin{tabular}{|c|l|c|}
\hline \multirow{2}{*}{ Sr.no } & \multicolumn{2}{|c|}{ Types of Disc Brake Rotor } \\
\cline { 2 - 3 } & \multicolumn{1}{|c|}{ Solid or Vented } & Application \\
\hline 1. & Drilled only & Street, all-weather rotor \\
\hline 2. & Blank \& Smooth & Street and light weight vehicles \\
\hline 3. & Slotted only & Street, Towing/Hauling, Off-Road \\
\hline 4. & Slotted or Grooved & Trucks and off-road vehicles. \\
\hline 5. & Dimpled & Sports car, weight reduction \\
\hline 6. & Waved & Race \& Sports vehicles \\
\hline
\end{tabular}

\section{A. Types of Disc Brakes}

Flat Disc Brakes (Blank and Smooth) have a large surface area which is in contact with brake pad and give excellent braking ability. But after prolonged braking periods it will lose its efficiency. Gases \& heat generated and also the particles of friction pad material can accumulate between disc and braking pad which will cause brake fading and thus wrapping the disc if the heat produced reaches the critical levels. It is used for typical driving conditions and are not expensive for manufacturing and machining.

Vented Disc Brake rotor are needed for dissipating excess heat through Vanes/Spokes present in between two discs. Due to large contact surface with fluid - air, the heat can easily dissipate by radiation via Vanes/Spokes. There is a slight increase in disc weight but with more durability and effective cooling of the disc for better performance. These Vanes/Spokes helps in better ventilation and thus prevents from cracking of the disc.

Drilled Disc Brake rotor are used due to its increased surface aera by the drilling number of holes in the disc. These drilled holes reduce the disc weight and increase the surface area to provide escape route for friction pad particles, heat and gases produced during braking. The drilled holes don't affect the structural integrity of the rotor and thus it is more effective for cooling the disc quickly. They are generally used for its looks and work better in wet conditions. 
Slotted or Grooved Disc brake rotor working is similar to the Drilled Disc brake rotor. The grooves are designed at angle in direction of rotation on the both sides of the disc. Due to its design, it allows gases to easily escape and increase friction on brake pad for better performance. But the grooves present generate noise during the scrubbing of brake pad. Also, it goes for its looks and are free from dirt and mud because of the grooves. Thus, they are used in off-road vehicles and trucks.

By using both grooves and holes in combination, they give a big advantage of the allowing debris (mud, dirt and friction pad particles) to escape and produce less noise during braking. This type of disc brake rotor work better in both dry and wet conditions and has extended life, better thermal dissipation and aesthetic looking. They are generally used in Sports and Race cars and are most expensive in the market.

Waved Edge Disc brake rotor is the latest design performance disc available for its less weight and improved cooling. Due to wavy edge design looks and its better performance than that of other discs, it is mostly picked by OEM and consumers.

\section{B. Disc Brake Problems}

Uneven Disc brake rotor wear due to excessive rotor distortion produces variations in thickness. When the brakes are applied, these variations can be felt as pedal pulsations which affects vehicle ride comfort. Hard spots generally are produced by sticky caliper or during casting of the disc brake rotor. These spots lead to variable thickness and thus produce pedal pulsations.

According to different vehicle applications, vehicle manufacturers have variations of rib configuration in the disc brake rotors. They currently use seventy different rib configurations in disc brake rotors. These are straight, vanned or segmented. The reason to use these many kind of rib configurations is to improve \& maximize cooling and make them less noisy and reduce brake squeal. Different design and variations in rib configuration can change the airflow and noise characteristics in the rotor.

Based upon braking application of the vehicle, the disc material needs to absorb \& disperse large amount of heat without braking performance being adversely affected [1].

Structural Analysis and Thermal analysis are a primordial stage in the study of the brake systems because the temperature determines thermo-mechanical behaviour of the structure [2].

The large amount of heat produced at the pad/disc interface during emergency braking indisputably evokes non-uniform temperature distributions in the domain of the rotor [2]. The energy dissipated in the form of heat can generate temperature ranging from $300^{\circ} \mathrm{C}$ to $800^{\circ} \mathrm{C}[2,3]$.

Braking performance of a vehicle can be significantly affected by the temperature rise in the brake components [4]. High temperatures during braking may cause brake fade, premature wear, brake fluid vaporization, bearing failure, thermal cracks and thermally-excited vibration [4]. Therefore, it is important to predict the temperature rise of a given brake system and assess its thermal performance in the early design stage [5].

\section{Material Analysis.}

Material Requirement for Disc Brake rotor: As based on the functionality and its possible defects, material properties are needed to take into consideration. Disc brake rotor material need high strength and high stiffness even at elevated temperatures because of high amount heat generated due to friction brake pad. It should have Low density and High thermal conductivity to easily dissipate heat. Also, the material needs Excellent abrasion resistance and Good creep resistance.

Most common material used in disc brake rotor is galvanized Cast Iron because of its high strength and low cost. Latest materials being used in these are MMC (Metal Matrix Composites). Generally, it is Aluminium Matrix Composites which is based on aluminium and silicon alloys and are most suitable for automobile disc brake rotor.

TABLE II. SCALED PROPERTIES OF MATERIALS

\begin{tabular}{|c|c|c|c|c|c|}
\hline \multirow{2}{*}{ Material } & \multicolumn{4}{|c|}{ Scaled Properties } \\
\cline { 2 - 6 } & $\begin{array}{c}\text { Compressive } \\
\text { Strength } \\
(\mathbf{M P a})\end{array}$ & $\begin{array}{c}\text { Friction } \\
\text { coefficien } \\
\boldsymbol{t}(\boldsymbol{\mu})\end{array}$ & $\begin{array}{c}\text { Wear } \\
\text { rate }\left(* 1 \mathbf{1 0}^{-}\right. \\
\mathbf{6} \mathbf{m m}^{3} \\
/ \mathbf{N m}^{-}\end{array}$ & $\begin{array}{c}\text { Performa } \\
\text { nce Index } \\
(\boldsymbol{\gamma})\end{array}$ & Rank \\
\hline GCI & 99 & 93 & 100 & 81.0 & 2 \\
\hline Ti-6Al-4V & 82 & 77 & 96 & 49.5 & 5 \\
\hline TMC & 100 & 70 & 29 & 56.0 & 4 \\
\hline AMC 1 & 31 & 80 & 73 & 79.0 & 3 \\
\hline AMC 2 & 59 & 100 & 81 & 88.6 & 1 \\
\hline
\end{tabular}

Table II. [7], shows the performance index of materials that can be used in automotive disc brake rotor. These materials are cast iron, titanium alloys \& reinforced composites and Aluminium matrix composites.

Many other materials like ceramic and carbon- carbon composites are used in high performance vehicles. These materials can withstand high temperatures and have great strength properties. Cost analysis of the disc brake rotor is to be done before finalizing design or material. The costliest material is the Ti-C reinforced composite (TMC). Alternate to Cast iron, there are relatively low cost AMC1 and AMC2 materials suitable to use in disc brake rotor.

\section{METHODOLOGY}

\section{A. Assumptions}

- The calculations done are considered for a light weight commercial vehicle.

- The dynamic weight transfer during braking on the front and rear axle is 60:40 percent of the total vehicle weight respectively.[5] 
- Ambient air-cooling is taken in to account along with forced convection where applicable with ambient temperature being constants.[5]

- The kinetic energy of the vehicle is lost in the form of thermal energy dissipated by the disc rotors.[5]

- The thermal conductivity of the material used for the analysis is uniform throughout.[6]

- Heat flux on each front wheel is applied on one side of the disc on the swept area, in a direction normal to the friction surface.[5]

- Radiative heat transfer is included in terms of an equivalent radiative heat transfer coefficient.[7]

TABLE III. VEHICLE SPECIFICATIONS

\begin{tabular}{|c|l|c|}
\hline \multirow{2}{*}{ Sr.no } & \multicolumn{2}{|c|}{ Light weight commercial vehicle } \\
\cline { 2 - 3 } & \multicolumn{1}{|c|}{ Parameters } & Values \\
\hline 1. & Mass of the Vehicle $(\mathrm{Kg})$ & 1400 \\
\hline 2. & Velocity of the Vehicle $\left(\mathrm{Kmph} / \mathrm{ms}^{-1}\right)$ & $120 / 33.33$ \\
\hline 3. & Input driver Force $(\mathrm{N})$ & 350 \\
\hline 4. & Pedal ratio & $5: 1$ \\
\hline 5. & Pedal Effort $(\mathrm{N})$ & 1750 \\
\hline 6. & Master Cylinder Bore Diameter $(\mathrm{mm})$ & 25 \\
\hline 7. & Disc Effective Radius $(\mathrm{mm})$ & 110 \\
\hline 8. & Caliper Piston Dia (mm) & 44 \\
\hline 9. & Friction Btw Rotor and Pad & 0.4 \\
\hline 10. & Friction Btw Road and tire & 0.7 \\
\hline 11. & Tire Radius (mm) & 406.4 \\
\hline
\end{tabular}

In this paper, we have considered single stop braking scenario where the vehicle is at $120 \mathrm{kmph}(33.33 \mathrm{~m} / \mathrm{s})$ and the brakes are applied to observe the structural and thermal behaviour of different types of Disc brake rotors. The disc brake material taken for analysis is Cast iron.

Some important properties of Gray Cast Iron are:

- Density - $7200 \mathrm{~kg} / \mathrm{m} 3$

- Young's Modulus - $125 \mathrm{GPa}$

- Thermal Conductivity $-54.5 \mathrm{~W} / \mathrm{mK}$

- Coefficient of Friction -0.25

We have performed static structural analysis and steady state thermal analysis of three types of Disc brake Rotors. These modules in ANSYS give the total deformation and temperature distribution of the disc after applying brakes at $120 \mathrm{kmph}(33.33 \mathrm{~m} / \mathrm{s})$ single Stop.

\section{B. Geometry}

Altering the geometry of the brake rotor is known to affect the total exposed area of the rotor, material content, and flow pattern of air and so on. Thus, geometric changes in turn are responsible for thermal stability and structural stability.
Our main focus here is to showcase the variation in structural stability and the temperature gradient of different types of brake rotors. A larger rotor moves faster through the pads, thereby generating more friction which indirectly provides better stopping power. More surface area provides more for heat dissipation, letting disc and pads running colder. Thickness of the rotor determines the maximum temperature it attains during braking and in important when it comes to keeping the brake components well in the operating temperature range.

In the considered scenario to avoid changes in the braking torque the effective radius and the thickness of all the models considered is kept the same.

\section{a. Solid Brake Rotor}

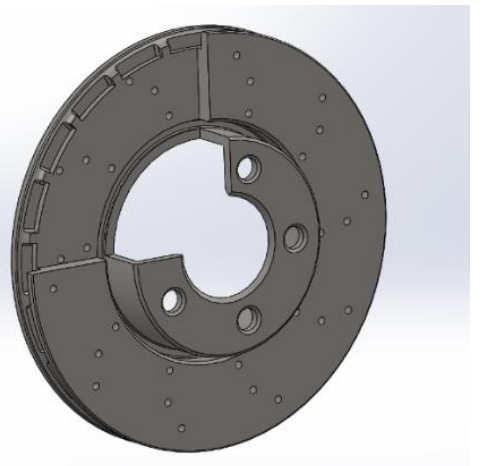

Fig. 1. Section View of a Drilled Solid Brake Rotor

TABLE IV. BRAKE ROTOR SPECIFICATIONS

\begin{tabular}{|c|l|c|}
\hline \multirow{2}{*}{ Sr.no } & \multicolumn{2}{|c|}{ Rotor Dimensions } \\
\cline { 2 - 3 } & \multicolumn{1}{|c|}{ Specification } & Value \\
\hline 1. & Inner Diameter $(\mathrm{mm})$ & 160 \\
\hline 2. & Outer Diameter $(\mathrm{mm})$ & 280 \\
\hline 3. & Height $(\mathrm{mm})$ & 47.33 \\
\hline 4. & Nominal Thickness $(\mathrm{mm})$ & 25 \\
\hline 5. & Minimum Thickness $(\mathrm{mm})$ & 7.5 \\
\hline 6. & Number of Holes & 30 \\
\hline 7. & Hub Register $(\mathrm{mm})$ & 68 \\
\hline 8. & Bolt Circle $(\mathrm{mm})$ & 53 \\
\hline 9. & Bolt Bore $(\varphi)(\mathrm{mm})$ & 15.3 \\
\hline 10. & Weight $(\mathrm{kg})$ & 8.26 \\
\hline
\end{tabular}

The cross drilled holes help in moving the air from the brake surface thus reducing the surface temperature. They also eliminate hot spots and warping of the rotor. Chamfering the holes and the various edges reduces the possibility of stress cracks. The optimum number of holes are drilled to provide the right compromise between the structural integrity and cooling. 


\section{b. Vanned Brake Rotor}

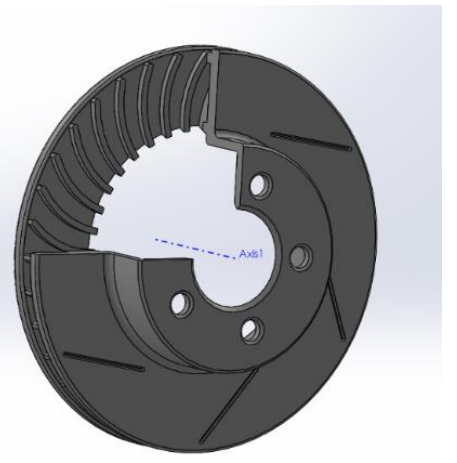

Fig. 2. Section View of a Vanned Brake Rotor

The area between successive vanes form a passage for air to flow. The number of vanes and the thickness of each element affects this area. A straight vane can be considered as a vane with infinite radius of curvature. Reducing this curvature effectively increases the available area and hence finds its use in a modern brake rotor design. The rounded slots on the brake surface sweep the gas and dust away from the wheels and clears the contact patch.

\begin{tabular}{|c|l|c|}
\multicolumn{2}{|c}{ TABLE V. $\quad$ BRAKE ROTOR SPECIFICATIONS } \\
\cline { 2 - 3 } Sr.no & \multicolumn{1}{|c|}{ Rotor Dimensions } \\
\cline { 2 - 3 } & \multicolumn{1}{|c|}{ Specification } & Value \\
\hline 1. & Inner Diameter $(\mathrm{mm})$ & 160 \\
\hline 2. & Outer Diameter $(\mathrm{mm})$ & 280 \\
\hline 3. & Height $(\mathrm{mm})$ & 67.33 \\
\hline 4. & Nominal Thickness $(\mathrm{mm})$ & 30 \\
\hline 5. & Minimum Thickness $(\mathrm{mm})$ & 10 \\
\hline 6. & Number of Vanes & 35 \\
\hline 7. & Hub Register $(\mathrm{mm})$ & 68 \\
\hline 8. & Bolt Circle $(\mathrm{mm})$ & 53 \\
\hline 9. & Bolt Bore $(\varphi)(\mathrm{mm})$ & 15.3 \\
\hline 10. & Weight $(\mathrm{kg})$ & 7.98 \\
\hline
\end{tabular}

Larger the Vane thickness, smaller the effective exposed area. This reduces the heat convention with air and increases the temperature of the disc. Vane height is varied directly with exposed weight and area.

\section{c. Diamond Pillared Brake Rotor}

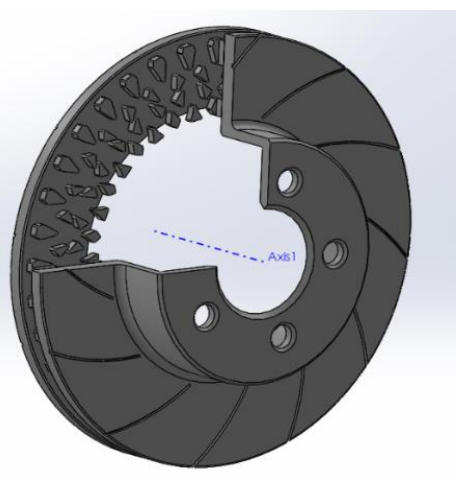

Fig. 3. Section View of a Diamond Pillared Brake Rotor
The patterned pillar structure improves the cooling efficiency of the brake rotor by increasing the overall contact area between air and the rotor body. This type of design also takes advantage of the centrifugal force to force air from the inside of the rotor to the outside. The various pillared structures create multiple air passages and give rise to turbulent flow. This induces a more efficient from of forced convective cooling lowering the maximum temperature reached by the component on prolonged breaking operation thus improving the durability.

TABLE VI. BRAKE ROTOR SPECIFICATIONS

\begin{tabular}{|c|l|c|}
\hline \multirow{2}{*}{ Sr.no } & \multicolumn{2}{|c|}{ Rotor Dimensions } \\
\cline { 2 - 3 } & \multicolumn{1}{|c|}{ Specification } & Value \\
\hline 1. & Inner Diameter $(\mathrm{mm})$ & 160 \\
\hline 2. & Outer Diameter $(\mathrm{mm})$ & 280 \\
\hline 3. & Height $(\mathrm{mm})$ & 67.33 \\
\hline 4. & Nominal Thickness $(\mathrm{mm})$ & 30 \\
\hline 5. & Minimum Thickness $(\mathrm{mm})$ & 10 \\
\hline 6. & Number of Pillar Elements & 144 \\
\hline 7. & Hub Register $(\mathrm{mm})$ & 68 \\
\hline 8. & Bolt Circle $(\mathrm{mm})$ & 53 \\
\hline 9. & Bolt Bore $(\varphi)(\mathrm{mm})$ & 15.3 \\
\hline 10. & Weight $(\mathrm{kg})$ & 7.85 \\
\hline
\end{tabular}

\section{Meshing}

Generating a mesh is an important step in the static structural and thermal analysis as the size of the finite element is determined by the mesh properties. Finer the mesh, more the accuracy of the results and more the computational power required. The ANSYS CFD Tetrameter uses unstructured meshing technology which follows an object-oriented approach. It produces the mesh directly from the CAD surfaces, and uses the Octree method to fill the volume with tetrahedral components. A robust smoothing algorithm ensures uniform consistency of the components.

The ANSYS CFD Tetrameter utilizes object-oriented unstructured meshing technology. It generates the mesh directly from the CAD surfaces and fills the volume with tetrahedral elements using the Octree approach. A robust smoothing algorithm provides uniform element quality.

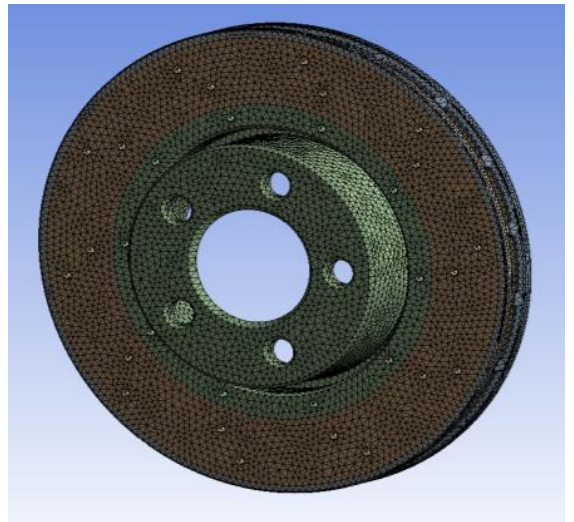

Fig. 4. The tetrahedral mesh generated for the Solid Brake Rotor 
Using the below fig. 5, mesh parameters 176,237 tetrahedral elements and 280,626 nodes are created. The Delaunay method which is employed by ANSYS CFD Tetrameter, is robust and fast; In addition, the Advancing Front method is its ability to generate a smoothly transitioning Tetra mesh with a controlled volume growth ratio.

\begin{tabular}{l|l|}
\hline \multicolumn{1}{l}{ Sizing } & \\
\hline Use Adaptive Sizing & Yes \\
\hline Resolution & Default (2) \\
\hline Mesh Defeaturing & Yes \\
\hline Defeature Size & Default \\
\hline Transition & Fast \\
\hline Span Angle Center & Coarse \\
\hline Initial Size Seed & Assembly \\
\hline Bounding Box Diagonal & $0.3988 \mathrm{~m}$ \\
\hline Average Surface Area & $5.1798 \mathrm{e}-004 \mathrm{~m}^{2}$ \\
\hline Minimum Edge Length & $2.5585 \mathrm{e}-005 \mathrm{~m}$ \\
\hline
\end{tabular}

Fig. 5. The mesh parameters for the above mesh

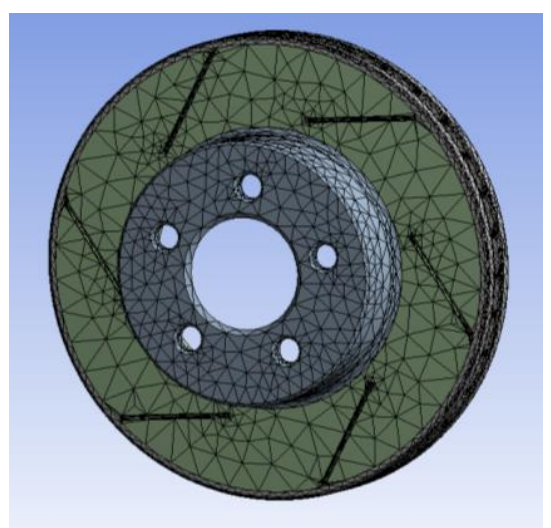

Fig. 6. The tetrahedral mesh generated for the Vanned Brake Rotor

\begin{tabular}{|l|l|}
$|l|$ \\
\hline Sizing \\
\hline Use Adaptive Sizi... & Yes \\
\hline Resolution & Default (2) \\
\hline Mesh Defeaturing & Yes \\
\hline$\square$ Defeature Size & Default \\
\hline Transition & Slow \\
\hline Span Angle Center & Medium \\
\hline Initial Size Seed & Assembly \\
\hline Bounding Box Di... & $0.40166 \mathrm{~m}$ \\
\hline Average Surface ... & $8.2856 \mathrm{e}-004 \mathrm{~m}^{2}$ \\
\hline Minimum Edge L... & 1. e-003 m \\
\hline
\end{tabular}

Fig. 7. The tetraahedral mesh generated for the Diamond Pilllared Brake Rotor

Using the above fig 7. mesh parameters 280,104 tetrahedral elements and 399,242 nodes are created.

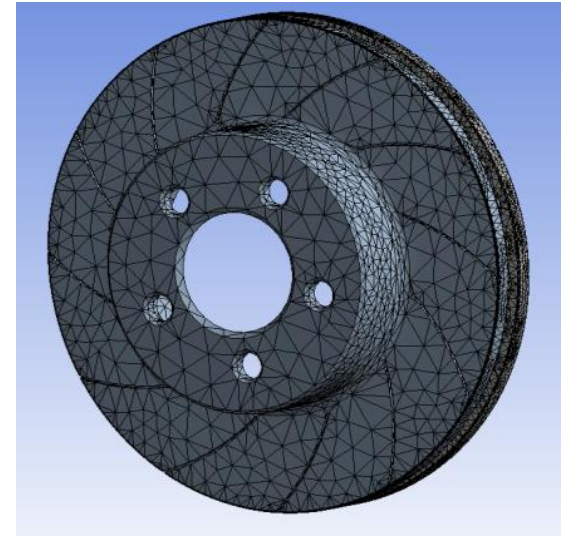

Fig. 8. The mesh parameters for the above mesh

\begin{tabular}{|l|l|}
\hline \multicolumn{2}{|l|}{ Sizing } \\
\hline Use Adaptive Sizi... & Yes \\
\hline Resolution & Default (2) \\
\hline Mesh Defeaturing & Yes \\
\hline$\square$ Defeature Size & Default \\
\hline Transition & Slow \\
\hline Span Angle Center & Medium \\
\hline Initial Size Seed & Assembly \\
\hline Bounding Box Di... & $0.40166 \mathrm{~m}$ \\
\hline Average Surface ... & $8.2856 \mathrm{e}-004 \mathrm{~m}^{2}$ \\
\hline Minimum Edge L... & $1 . \mathrm{e}-003 \mathrm{~m}$ \\
\hline
\end{tabular}

Fig. 9. The mesh parameters for the above mesh

Similarly using the previous fig 7. mesh parameters 291,254 tetrahedral elements and 414,356 nodes are created.

\section{Boundary Conditions}

The required parameters for calculations and results are the following below:

- $\quad \mathrm{K} . \mathrm{E}=777622.33$ Joule

- Deceleration of the vehicle $=6.86 \mathrm{~ms}^{-2}$

- $\quad$ Time taken to stop the vehicle $=4.853 \mathrm{sec}$

- Total Stopping distance $=131.03 \mathrm{~m}($ Reaction time $=$ $1.5 \mathrm{~s})$

- $\quad$ Braking torque on disc $=354.42 \mathrm{Nm}$

- $\quad$ Clamping force $=2301.42 \mathrm{~N}$

- $\quad$ Heat flux $=48.58 \mathrm{~kW} / \mathrm{m}^{2}$

- Convention (heat transfer coefficient $)=250 \mathrm{~W} / \mathrm{m}^{2} \mathrm{C}$ 


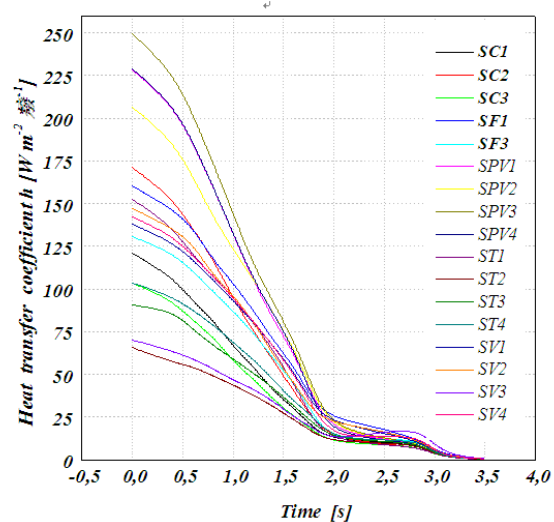

Fig. 10. Heat transfer coefficient for Convention

Applying the boundary conditions is the next step in FEA. Since we plan on solving the model for Total Deformation and Temperature Gradient two separate setups are uses with separate boundary conditions.

Based on the various calculation carried out, a frictional force of $2301.1 \mathrm{~N}$ is applied on the both contact faces of the rotor. Rotational velocity is also imparted to the entire body. When the brake pads are actuated and pressed on the rotor braking torque is exerted on each face of the rotor which is represented by a moment of $0.35 \mathrm{~N}-\mathrm{m}$ on each face in the respective directions as shown above.

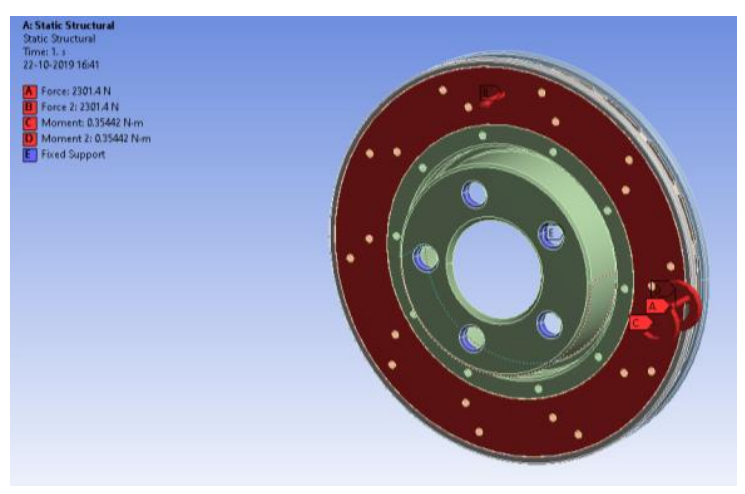

Fig. 11. The forces and the moments applied to the Solid Brake Rotor

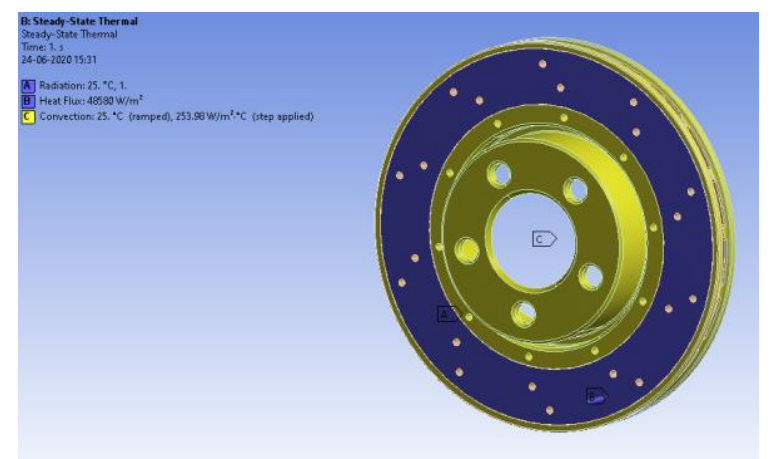

Fig. 12. Various Convective and Radiative surfacesa of the Solid Brake Rotor

For Thermal Analysis all the surfaces of the rotor exposed to the atmosphere are considered for forced convective heat transfer. The ambient temperature is taken as $25^{\circ} \mathrm{C}$ Since we are considering a single stop braking scenario majority of the heat is generated due to the friction between the pads and the contact patch. Thus, heat transfer through radiation will be prominent in this area when compared to the rest of the rotor.

Similarly, the above-mentioned boundary conditions are applied accordingly to other brake rotors in consideration.

\section{RESULTS}

The FEA Analysis provides is with:

- Total Deformation

- Temperature Gradient

The achieved results are compiled below.

By drilling holes, the effective area exposed to air increases thus increasing heat dissipation through convective heat transfer. This is achieved without any detrimental effect on the structural integrity of the brake disc as seen in the Total Deformation infographic above. This design can be potentially implemented in an automobile with further testing. Drilling holes also decreases the mass of the rotor which can in turn reduce the unsprung mass for the vehicle. This provides an opportunity to use a less expensive and less rigid suspension for improved ride quality and passenger comfort as well.

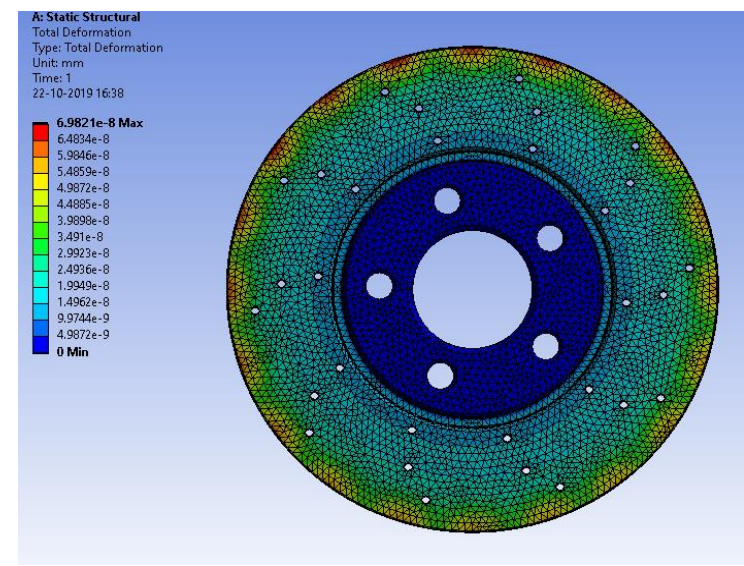

Fig. 13. Total deformation as seen in the Solid Brake Rotor

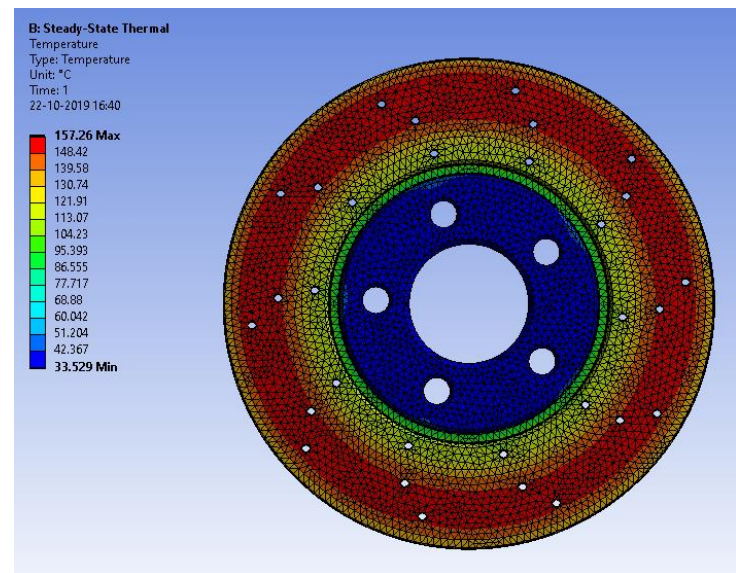

Fig. 14. Temperature distribution across the Solid Brake Rotor 


\section{A. Vanned Brake Rotor}

The curved vanes create a relatively low-pressure zone inside the rotor as it spins by throwing out air using centrifugal force. This action increases with the speed of the rotor thus improving the cooling efficiency. However, it can be seen that in comparison to the drilled solid rotor the temperatures are slightly in the higher range, this is primarily due to lesser surface are for heat dissipation in the vanned rotor. Structural integrity of this brake rotor is also not compromised.

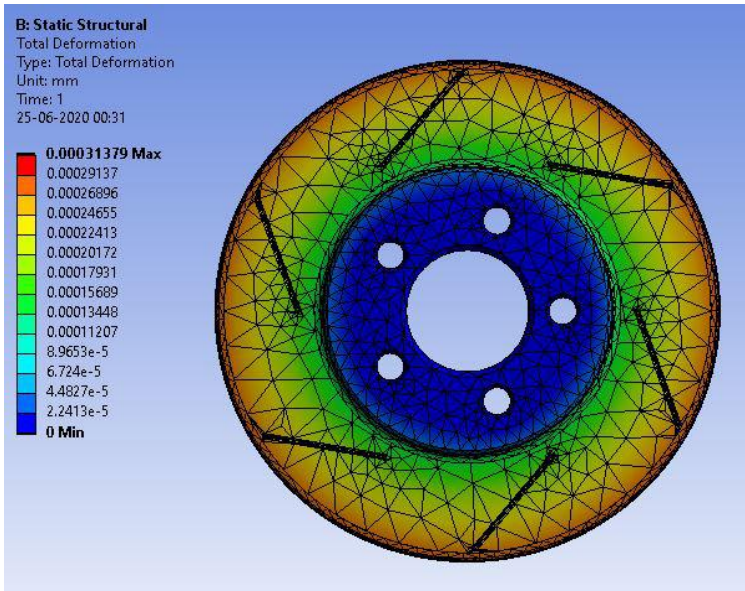

Fig. 15. Total deformation as seen in the Vanned Brake Rotor

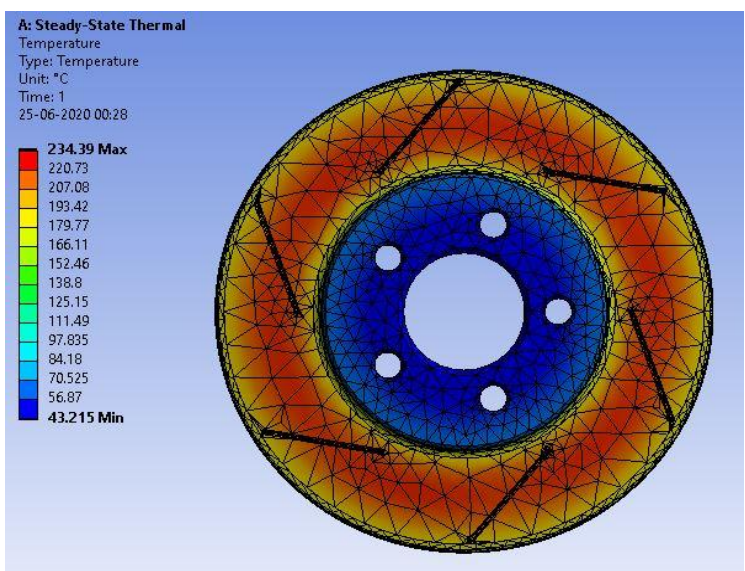

Fig. 16. Temperature distribution across the Vanned Brake Rotor

The diamond pillared structure provides with a more uniform mass flow rate of air thus increasing the heat dissipation even though the actual mass flow rate is comparatively lower than its vanned counterpart. The temperature distribution is also more uniform and symmetrical leading to lower temperature gradients across the rotor surface. The reduction in weight is another benefit of this design. Overall, we can observe that the Diamond Pillared ventilation system provides the best cooling performance while maintaining good structural integrity and hence presents itself as a promising design over the other more common brake rotors.

\section{B. Diamond Pillared Brake Rotor}

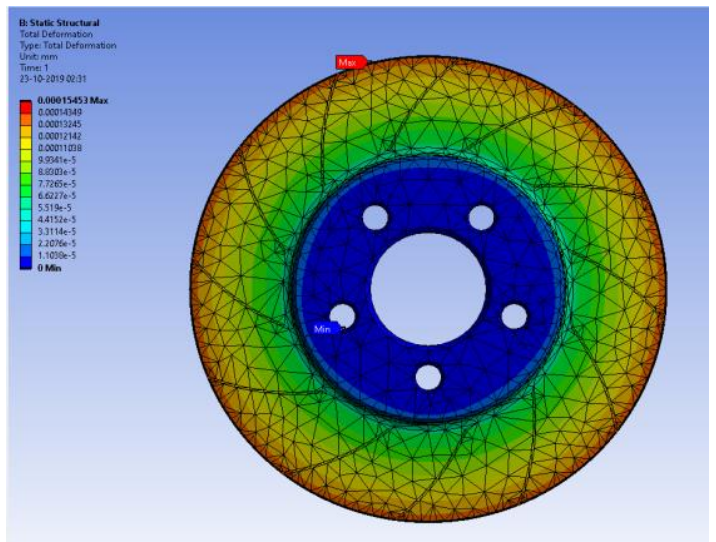

Fig. 17. Total deformation as seen in the Diamond Pillared Brake Rotor

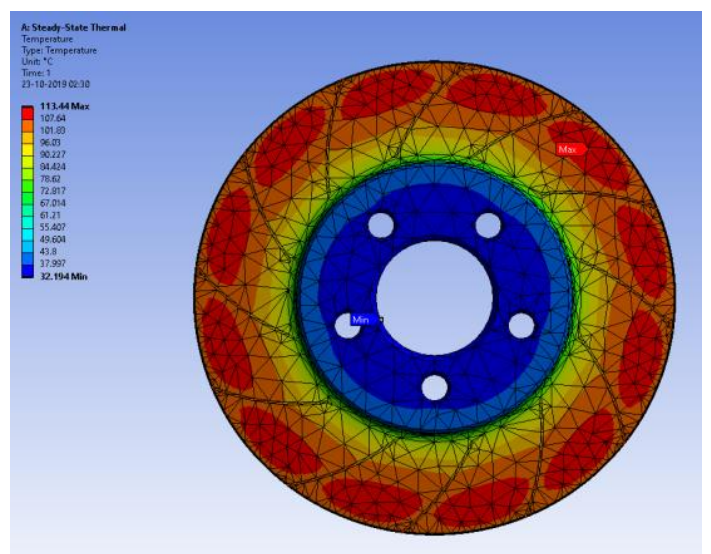

Fig. 18. Temperature distribution across the Diamond Pillared Brake Rotor

\section{DISCUSSION}

Higher the total surface area of the rotor exposed to air; higher the heat transfer occurs. But larger outer diameter of disc will increase total weight of rotor even though the surface area is increased. That's why different types of design such as vanes or kangaroo is used between the two discs to increase the total surface area and hence increasing heat transfer by convention. In Vane Disc Brake rotor, the thickness is as minimum as possible to increase the air flow passage. Larger vane thickness increase weight and reduce air flow in the disc. Based on the results, the vane disc brake's structural integrity is good but the temperature reached is higher. This can affect the braking performance and causes problems. Curved Vanes are used in case of small outer diameter and have greater heat dissipation capacity than straight vanes. Its complexity results in higher cost of manufacturing and used in high performance vehicles. This type of disc rotor can be used in real time as the deformation is very low as $0.00031379 \mathrm{~mm}$.

When compared to vane disc brake rotor, Solid disc brake shows better results in temperature distribution. The drilled holes in solid disc brake reduces the weight and improve selfcleaning. This type is available in low cost compared to curved vane brake disc. The total deformation of solid brake rotor is very much low $0.0000698 \mathrm{~mm}$. For single stop, the maximum temperature of solid type reached is $157.260 \mathrm{C}$. 
Finally, the Diamond paw/pillar design has the most effective cooling as it has greater flow of air and a larger total surface area exposed to the air. This type of brake rotor can also be used in real time because its total deformation is $0.0001543 \mathrm{~mm}$. The grooves present on this disc surface helps in improving braking performance by increasing the friction. These grooves also help in removing the dust, dirt and debris. Drilling holes in pillar design can affect its structural integrity and cannot be used in real time. The temperature distribution in pillar design is better than both solid and vanned disc and the maximum temperature reached is $113.440 \mathrm{C}$.

\section{CONCLUSION}

Material and Disc brake geometry depend on the specifications of vehicle and braking force needed. Discs brake rotors with different geometric patterns (holes and slots/Grooves) and different vented designs between the discs results in weight reduction and faster cooling during braking. These variations also have impact on its lifespan. For Pillar/Kangaroo and Vane type rotor, due to their design more turbulence is created and has higher mass flow rate of air to provide better convention for cooling. As their design is complex, they are expensive for production.

\section{FUTURE SCOPE}

This paper presents the benefits of the pillared rotor design which with adequate dimensional variation and practical testing can provide reliable braking performance of various commercial vehicle. This is design can be further refined by conducting extensive material testing and making the adequate variations to the various dimensions like the pillar shape, thickness and so on. The current challenge for this rotor design is the high cost of manufacturing with the present technology, thus providing opportunities for innovation in manufacturing technique in the future.

\section{REFERENCES}

[1] Fred Puhn, "Brake Handbook" (HP Books, U.S.A., 1985), ISBN: 089586-232-8

[2] Ali Belhocine, Mostefa Bouchetara, "Thermal analysis of solid brake disc", Elsevier, Applied Thermal Engineering 32 (2012)59-67, 2012. doi : 10.1016 /j.applthermaleng. 2011.08.029

[3] Rudolf Limpert, "Brake Design and Safety" (S.A.E. Internationl, U.S.A., 1999), ISBN: 1-56091-915-9.

[4] Daanvir Karan Dhir, "Thermo-mechanical performance of automotive disc brakes", Materials Today: Proceedings 5 (2018).

[5] Aleskander Yevtushkendo, Michal Kuciej, "Temperature and thermal Stresses in a pad/disc during braking", Elsevier, Applied Thermal Engineering 30 (2010) 354-359,2011.

[6] Guru Murthy Nathi, T N Charyulu, K.Gowtham, P. Satish Reddy, "Coupled Structural/ Thermal Analysis of Disc Brake", IJERT: 539553, 2012.

[7] M.A. Maleque ${ }^{1}$, S.Dyuti 2 and M.M. Rahman, Material Selection Method in Design of Automotive Brake Disc, WCE 2010 ,SBN: 978988-18210-8-9 ISSN: 2078-0966. 